\title{
Generation of entanglement in regular systems
}

\author{
Marko Žnidarič and Tomaž Prosen \\ Physics Department, Faculty of Mathematics and Physics, University of Ljubljana, Ljubljana, Slovenia
}

(Dated: November 12, 2018)

\begin{abstract}
We study dynamical generation of entanglement in bipartite quantum systems, characterized by purity (or linear entropy), and caused by the coupling between the two subsystems. Explicit semiclassical theory of purity decay is derived for integrable classical dynamics of the uncoupled system, and for localized (general Gaussian wave-packet) initial states. Purity decays as an algebraic function of time $\times$ strength of perturbation, independently of the Planck's constant.
\end{abstract}

PACS numbers: 3.65.Ud, 3.67.Mn, 3.65.Sq

Detailed understanding of entanglement, being one of the most distinct features of the quantum world, is an issue of high importance, particularly in view of recent efforts to build quantum devices that will manipulate (pure states of) individual quantum systems. The loss of control over the entanglement, e.g. decoherence, in such a device is one of the major obstacles that we have to overcome.

In the present paper we are going to study dynamical generation of entanglement in bipartite systems. Initially separable pure state will get entangled due to the coupling between two subsystems. Here we consider systems where the uncoupled part of the Hamiltonian in both subsystems generates regular (integrable) dynamics in the classical limit. The motivation to study entanglement generation in systems with a regular uncoupled dynamics comes from the fact that such systems are quite common both in experiments and as theoretical models. For instance, if the uncoupled system consists of a number of uncoupled one degree of freedom (DOF) systems then it is integrable. Such is the case in various proposals for quantum computation, e.g. ion traps. Further, experimentally realizable Jaynes-Cummings model, where decoherence for cat states [1] has actually been experimentally measured, is also an integrable system. Still further, a standard model of decoherence [2] consists of an infinite number of harmonic oscillators. If the bath consists of a finite number of harmonic oscillators this falls under the domain of our theory. Recently [3] it has been pointed out that the decoherence for truly macroscopic superposition is so fast that the usual master equation approach is not valid anymore. On this very short "instantaneous" time scale any system will effectively behave as a regular one (i.e. correlations do not decay yet). The semiclassical decay of purity has recently been discussed by Jacquod [4], however his results in the regular domain do not agree with our findings. See [5] for some other related recent results.

Time evolution of the system will be governed by a Hamiltonian

$$
H=H_{0}+\delta \cdot V, \quad H_{0}=H_{\mathrm{A}} \otimes \mathbb{1}_{\mathrm{B}}+\mathbb{1}_{\mathrm{A}} \otimes H_{\mathrm{B}},
$$

where $H_{0}$ is an uncoupled part of the Hamiltonian and $V$ is the coupling between the two subsystems responsible for the generation of entanglement. The strength of this coupling is given by a dimensionless parameter $\delta$. We will use subscripts "A" and "B" to denote two subsystems. The state of the whole system at time $t$ is simply $|\psi(t)\rangle=U(t)|\psi(0)\rangle$, with a unitary propagator $U(t)=\exp (-\mathrm{i} H t / \hbar)$. Let us define time-averaged coupling

$$
\bar{V}=\lim _{T \rightarrow \infty} \frac{1}{T} \int_{0}^{T} d t V(t)
$$

where $V(t)$ is the coupling operator in the interaction picture, $V(t)=U_{0}^{\dagger}(t) V U_{0}(t), U_{0}(t)=\exp \left(-\mathrm{i} H_{0} t / \hbar\right)$, i.e. propagated with an uncoupled part of the Hamiltonian. We shall assume a situation, typical for a regular $H_{0}$, where $\bar{V}$ is a non-trivial operator, different from zero or a multiple of identity [ 6 ]. We wish to stress that perturbation $V$ will typically break the integrability of $H$, and that our results reported below are not limited to values of small $\delta$.

The entanglement between the two subsystems, for a pure state $|\psi(t)\rangle$, is characterized by a purity

$$
I(t)=\operatorname{tr}_{\mathrm{A}}\left[\rho_{\mathrm{A}}^{2}(t)\right], \quad \rho_{\mathrm{A}}(t)=\operatorname{tr}_{\mathrm{B}}[\rho(t)]
$$

where $\rho(t):=|\psi(t)\rangle\langle\psi(t)|$. Iff purity $I(t)$ is less than 1 , the two subsystems are entangled, otherwise they are in a separable (product) state. Our initial state will always be a product one, $|\psi(0)\rangle=\left|\psi_{\mathrm{A}}(0)\right\rangle \otimes\left|\psi_{\mathrm{B}}(0)\right\rangle$, hence $I(0)=$ 1. The initial states $\left|\psi_{\mathrm{A}, \mathrm{B}}(0)\right\rangle$ will be Gaussian wave packets. Time dependence of purity $I(t)$ will then tell us how fast the two subsystems get entangled due to the coupling $V$.

Let us proceed with the calculation of purity decay $I(t)$. We should observe that propagating the state backwards in time with a separable - uncoupled dynamics $U_{0}(t)$ does not change the value of purity, so $\rho(t)$ in Eq. (3) can be replaced by

$$
\rho^{\mathrm{M}}(t)=M(t) \rho(0) M^{\dagger}(t), \quad M(t)=U_{0}^{\dagger}(t) U(t),
$$

where $M(t)$ is the echo operator used in the theory of fidelity decay [7, [8]. The matrix $\rho^{\mathrm{M}}(t)$ represents the evolution of our pure state in the interaction picture. As just explained above, the purity (3) is equal to

$$
I(t)=\operatorname{tr}_{\mathrm{A}}\left[\left\{\rho_{\mathrm{A}}^{\mathrm{M}}(t)\right\}^{2}\right], \quad \rho_{\mathrm{A}}^{\mathrm{M}}(t)=\operatorname{tr}_{\mathrm{B}}\left[\rho^{\mathrm{M}}(t)\right] .
$$


An advantage of the representation (5) over (3) is the fact that the echo operator $M_{\delta}(t)$ is, unlike the forward evolution $U(t)$, close to an identity for small $\delta$ so one may use perturbative, or asymptotic expansions in $\delta$. We follow the approach of Ref. 7] and use the Baker-CampbellHausdorff formula $e^{\delta V} e^{\delta W}=\exp \left(\delta(V+W)+\frac{1}{2} \delta^{2}[V, W]+\right.$ ...) for continuous products, see e.g. [9], to simplify the expression for the echo operator $M(t)$. The lowest order term in the exponential is $\frac{\delta}{\hbar} \int d t V(t)$. For times larger than some classical averaging time $t_{\text {ave }}$, on which $\bar{V}$ (2) converges, this term can be rewritten as $\frac{\delta}{\hbar} \bar{V} t$. The second order term in $\delta$ can be shown to grow with time no faster than $\delta^{2} t / \hbar$, and by induction higher orders can be estimated to grow as $\sim \delta^{r} t^{r-1} / \hbar$. Therefore, provided only $\delta \ll 1$, higher orders in $\delta$ can be neglected and we end up with a very simple expression for the echo operator

$$
M(t)=\mathrm{e}^{-\mathrm{i} \delta t \bar{V} / \hbar} .
$$

So the echo operator can be interpreted as the propagator with an effective Hamiltonian $\delta \bar{V}$. We proceed with a semiclassical evaluation of the purity, a procedure completely analogous to a similar calculation for the fidelity [10]. We use the notation in which small Latin letters denote classical limiting observables (e.g. Weyl symbols) of the corresponding operators denoted by capital Latin letters. For example, let $\mathbf{j}=\left(\mathbf{j}_{\mathrm{A}}, \mathbf{j}_{\mathrm{B}}\right)$ denote a $d=d_{\mathrm{A}}+d_{\mathrm{B}}$ dimensional vector of classical canonical actions of the completely integrable uncoupled classical Hamiltonian $h_{0}=h_{\mathrm{A}}+h_{\mathrm{B}} \cdot d_{\mathrm{A}}$ and $d_{\mathrm{B}}$ are the number of DOF of the subsystems "A" and "B", respectively. In quantum mechanics, one has a vector of mutually commuting action operators $\mathbf{J}$, with a common set of eigenvectors, denoted by a multi-index $\mathbf{n} \in \mathbb{Z}^{d}$ of quantum numbers: $\mathbf{J}|\mathbf{n}\rangle=\hbar(\mathbf{n}+\boldsymbol{\alpha})|\mathbf{n}\rangle \approx \hbar \mathbf{n}|\mathbf{n}\rangle$ where $\boldsymbol{\alpha}$ are the Maslov indices. Here and below " $\approx$ " means equal in the leading order in $\hbar$. The purity (5D) can now be written as a sum over $d$-dimensional lattice of quantum numbers, using the fact since $\bar{V}$ commutes with $H_{0}$ it is diagonal in the basis $|\mathbf{n}\rangle$, and in the leading semiclassical order (in $\hbar)$ we can replace the summation by an integral over the classical action space. Further, we replace the operator $\bar{V}$ by its classical limit $\bar{v}(\mathbf{j})$, which is a conserved quantity so it is a function of $d$ classical actions $\mathbf{j}$ only. Let us denote by $p(\mathbf{j})=p_{\mathrm{A}}\left(\mathbf{j}_{\mathrm{A}}\right) p_{\mathrm{B}}\left(\mathbf{j}_{\mathrm{B}}\right)$ the classical limit of the initial density $\langle\mathbf{n}|\rho(0)| \mathbf{n}\rangle$. For our initial product state of two wave-packets each of the two densities is a Gaussian

$$
p_{\mathrm{a}}\left(\mathbf{j}_{\mathrm{a}}\right)=C \exp \left\{-\left(\mathbf{j}_{\mathrm{a}}-\mathbf{j}_{\mathrm{a}}^{*}\right) \Lambda_{\mathrm{a}}\left(\mathbf{j}_{\mathrm{a}}-\mathbf{j}_{\mathrm{a}}^{*}\right) / \hbar\right\},
$$

where a subscript "a" takes values "A" or "B", depending on the subsystem, $\mathbf{j}_{\mathbf{a}}^{*}$ is the position of the initial packet, $\Lambda_{\mathrm{a}}$ is a positive squeezing matrix and $C=(\hbar / \pi)^{d_{\mathrm{a}} / 2} \sqrt{\operatorname{det} \Lambda_{\mathrm{a}}}$ is normalization constant. The purity can now be written as an integral

$$
\begin{array}{r}
I(t) \approx \hbar^{-2 d} \int d \mathbf{j} d \tilde{\mathbf{j}} \exp \left(-\mathrm{i} \frac{\delta t}{\hbar} \Phi\right) p(\mathbf{j}) p(\tilde{\mathbf{j}}), \\
\Phi=\bar{v}\left(\mathbf{j}_{\mathrm{A}}, \mathbf{j}_{\mathrm{B}}\right)-\bar{v}\left(\tilde{\mathbf{j}}_{\mathrm{A}}, \mathbf{j}_{\mathrm{B}}\right)+\bar{v}\left(\tilde{\mathbf{j}}_{\mathrm{A}}, \tilde{\mathbf{j}}_{\mathrm{B}}\right)-\bar{v}\left(\mathbf{j}_{\mathrm{A}}, \tilde{\mathbf{j}}_{\mathrm{B}}\right) .
\end{array}
$$

Next we expand the phase $\Phi$ around the position $\mathbf{j}^{*}=$ $\left(\mathbf{j}_{\mathrm{A}}^{*}, \mathbf{j}_{\mathrm{B}}^{*}\right)$ of the initial packet. The constant and the linear terms cancel exactly and the lowest order non-vanishing term is quadratic

$$
\Phi \approx\left(\mathbf{j}_{\mathrm{A}}-\tilde{\mathbf{j}}_{\mathrm{A}}\right) \cdot \bar{v}_{\mathrm{AB}}^{\prime \prime}\left(\mathbf{j}^{*}\right)\left(\mathbf{j}_{\mathrm{B}}-\tilde{\mathbf{j}}_{\mathrm{B}}\right)+\cdots,
$$

where $\bar{v}_{\mathrm{AB}}^{\prime \prime}$ is a $d_{\mathrm{A}} \times d_{\mathrm{B}}$ matrix of mixed second derivatives of $\bar{v}$ evaluated at the position of the initial packet,

$$
\left(\bar{v}_{\mathrm{AB}}^{\prime \prime}\right)_{k l}=\frac{\partial^{2} \bar{v}}{\partial\left(\mathbf{j}_{\mathrm{A}}\right)_{k} \partial\left(\mathbf{j}_{\mathrm{B}}\right)_{l}} .
$$

Using this expansion in the integral for purity we see that the resulting $2 d$ dimensional integral is Gaussian and can therefore be expressed in terms of a determinant of a $2 d \times 2 d$ matrix. Using special properties of the resulting matrix the determinant can be reduced [1] to a determinant of a $d_{\mathrm{A}} \times d_{\mathrm{A}}$ matrix, with the final result

$$
I(t)=\frac{1}{\sqrt{\operatorname{det}\left(\mathbb{1}+(\delta t)^{2} u\right)}}, \quad u=\Lambda_{\mathrm{A}}^{-1} \bar{v}_{\mathrm{AB}}^{\prime \prime} \Lambda_{\mathrm{B}}^{-1} \bar{v}_{\mathrm{BA}}^{\prime \prime},
$$

where $u$ is a $d_{\mathrm{A}} \times d_{\mathrm{A}}$ matrix involving $\bar{v}_{\mathrm{AB}}^{\prime \prime}$ and its transpose $\bar{v}_{\mathrm{BA}}^{\prime \prime}$. Note that the matrix $u$ is a classical quantity (independent of $\hbar$ ) that depends only on observable $\bar{v}$ and on the position of the initial packet. This explicit formula for purity decay is the main result of the present paper [15].

Before discussing its consequences let us remind on its range of validity. The restrictions are rather weak: $\bar{v}$ must be nonvanishing (typical for regular systems) and smooth on a scale of the initial packet $\propto \sqrt{\hbar}$, time must be larger than the averaging time $t>t_{\text {ave }}$, and the coupling must be small $\delta<1$. In addition, the phase $\Phi$ should increment by a small amount for neighboring quantum numbers, which translates into the condition $\delta t|| \bar{v}_{\mathrm{AB}}^{\prime \prime} \|<1 / \hbar$.

The most prominent feature of the formula (11) for the purity decay for initial product wave packets is its $\hbar$ independence. In the linear response calculation this $\hbar$-independence has already been theoretically predicted [12] as well as numerically confirmed [13]. Here we have a full expression to all orders. We also see that the scaling of the decay time $t_{\mathrm{d}}$ on which $I(t)$ decays is $t_{\mathrm{d}} \sim 1 / \delta$. This means that the purity will decay on a very long time scale and so the wave packets are universal pointer states [14], i.e. the most robust states. For small $\delta t$ we can expand the determinant and we get initial quadratic decay $I(t)=1-\frac{1}{2}(\delta t)^{2} \operatorname{tr}[u]+\cdots$. For large times we use the fact that $\operatorname{det}(\mathbb{1}+z u)$ is a polynomial in $z$ of order $r=\operatorname{rank}(u)$, so we have asymptotic power law decay $I(t) \asymp$ const $(\delta t)^{-r}$. Note that the rank of $u$ is bounded by the minimal of the subspace dimensions, i.e. $1 \leq r \leq \min \left\{d_{\mathrm{A}}, d_{\mathrm{B}}\right\}$, since the definition (3) is symmetric with respect to interchanging the roles of the subspaces "A" and "B". Let us give two simple examples: (i) For $d_{\mathrm{A}}=1$ and for any $d_{\mathrm{B}}$ we will always have asymptotic power law decay with $r=1$. If a single 
DOF of the subsystem "A" is coupled with all DOF of the subsystem "B", e.g. $\bar{v}=j_{\mathrm{A}} \otimes\left(j_{\mathrm{B} 1}+j_{\mathrm{B} 2}+\cdots\right)$, then $\left|\bar{v}^{\prime \prime}\right|^{2} \propto d_{\mathrm{B}}$ and we have $I(t) \asymp 1 /\left(\delta t \sqrt{d_{\mathrm{B}}}\right)$; (ii) Let us consider a multidimensional system where the matrix $u$ is of rank one so it can be written as a direct product of two vectors, $u=\mathbf{x} \otimes \mathbf{y}$. The determinant occurring in $I(t)$ is then simply $\operatorname{det}\left(\mathbb{1}+(\delta t)^{2} u\right)=1+(\delta t)^{2} \mathbf{x} \cdot \mathbf{y}$. Such is the case for instance if we have a coupling of the same strength between all pairs of DOF. The dot product is in this case $\mathbf{x} \cdot \mathbf{y} \propto d_{\mathrm{A}} d_{\mathrm{B}}$ and we have $I(t) \asymp 1 /\left(\delta t \sqrt{d_{\mathrm{A}} d_{\mathrm{B}}}\right)$, i.e. the power of the algebraic decay is independent of both $d_{\mathrm{A}}$ and $d_{\mathrm{B}}$.

In [4] the author predicted a universal asymptotic $t^{-2}$ decay of purity independent of the dimensions or the coupling involved. He obtained this result for an average purity, i.e. averaged over the position of the initial packet. Our result for $I(t)$ (11) clearly can not reproduce the result of Ref. [4], even if we average over the position of the initial packet. Such an average decay will in general depend on the functional dependence of the matrix $u$ on the position of the initial packet.

We continue with a numerical demonstration of the theoretical prediction for purity decay (11). For the first example we take a $1+1$ DOF system, $d_{\mathrm{A}}=d_{\mathrm{B}}=1$, of two an-harmonic oscillators with the uncoupled Hamiltonian

$$
H_{0}=\gamma_{\mathrm{A}}\left(\hbar a_{\mathrm{A}}^{+} a_{\mathrm{A}}-\Delta\right)^{2}+\gamma_{\mathrm{B}}\left(\hbar a_{\mathrm{B}}^{+} a_{\mathrm{B}}-\Delta\right)^{2},
$$

where $a^{+}, a$ are standard boson raising/lowering operators. For the coupling we take

$$
V=\hbar^{2}\left(a_{\mathrm{A}}^{+}+a_{\mathrm{A}}\right)^{2}\left(a_{\mathrm{B}}^{+}+a_{\mathrm{B}}\right)^{2} .
$$

The corresponding classical Hamiltonian $h$ reads

$h=\gamma_{\mathrm{A}}\left(j_{\mathrm{A}}-\Delta\right)^{2}+\gamma_{\mathrm{B}}\left(j_{\mathrm{B}}-\Delta\right)^{2}+16 \delta j_{\mathrm{A}} j_{\mathrm{B}} \sin ^{2} \theta_{\mathrm{A}} \sin ^{2} \theta_{\mathrm{B}}$

where $\theta_{\mathrm{a}}$ are the canonical angles. The initial wave packet on both subsystems is a boson coherent state

$$
\left|\psi_{\mathrm{A}}(0)\right\rangle=\left|\psi_{\mathrm{B}}(0)\right\rangle=|\alpha\rangle=\mathrm{e}^{\alpha a^{+}-\alpha^{*} a}|0\rangle,
$$

where $|0\rangle$ is the ground state. The parameter $\alpha$ is chosen as $\alpha=\sqrt{j^{*} / \hbar}$ with $j^{*}=0.1$. The squeezing parameter for the coherent states (15) is $\Lambda_{\mathrm{A}, \mathrm{B}}=1 /\left(2 j^{*}\right)$. Other parameters of the Hamiltonian are $\gamma_{\mathrm{A}}=1, \gamma_{\mathrm{B}}=0.6456$. The offset $\Delta=1.2$ was chosen in order to have nonzero classical frequency $\partial h / \partial j$ at the position of the initial packet. This is needed in order for $\bar{v}$ to be well defined. Time averaged coupling is calculated easily, $\bar{v}=4 j_{\mathrm{A}} j_{\mathrm{B}}$. The matrix $u$ is now just a number, $u=\left(8 j^{*}\right)^{2}$. Theoretical prediction for the purity decay is thus

$$
I(t)=\frac{1}{\sqrt{1+\left(8 j^{*} \delta t\right)^{2}}} .
$$

The results of numerical simulation together with the theory are shown in Figs. 1 and 2] In Fig. 11 we see

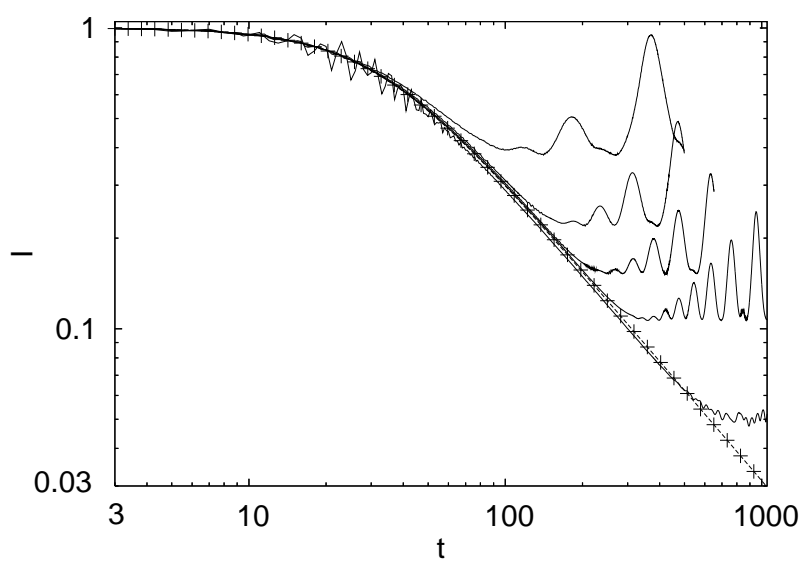

FIG. 1: Purity decay for $1+1$ DOF system (1213) for $\delta=0.04$ and different $1 / \hbar=10,25,50,100,500$, from top to bottom. Dashed line with pluses is the theoretical formula (16).

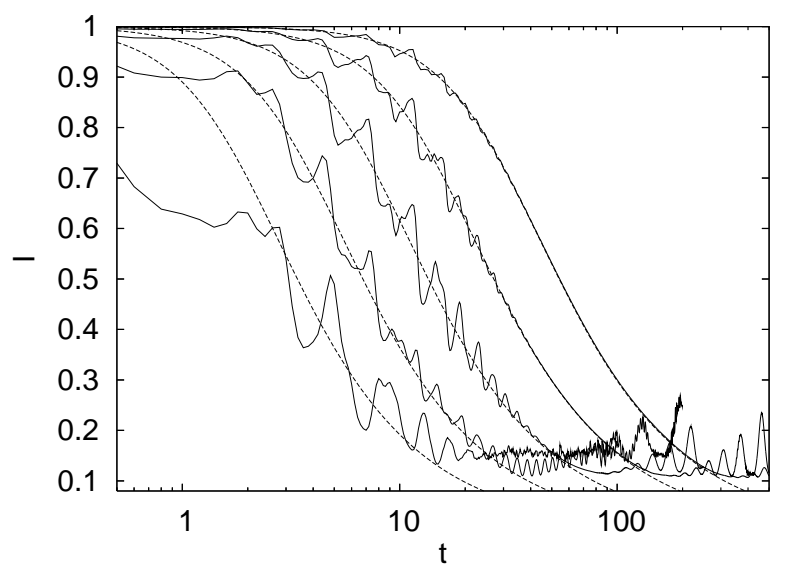

FIG. 2: Purity decay for $1+1$ DOF system (1213) for $\hbar=$ $1 / 100$ and different $\delta=0.64,0.32,0.16,0.08,0.04$, from left to right. Dashed lines give the theoretical prediction (16).

that the decay is indeed $\hbar$-independent, apart from a finite size fluctuating plateau after long time. The size of this plateau is of the order $I(t \rightarrow \infty) \sim 1 / N_{\text {eff }}$, where $N_{\text {eff }} \sim \sqrt{8 j^{*} / \hbar}$ is an effective Hilbert space dimension, i.e. the number of action eigenstates overlaping with the initial coherent state (15). Strong revivals for large $\hbar$ are a consequence of small number of available states $N_{\text {eff }}$ and low dimensionality. Revivals are expected to be less pronounced for larger dimensionalities $d_{\mathrm{A}}, d_{\mathrm{B}}$, similarly as for the fidelity [10]. For large times one can clearly observe asymptotic $t^{-1}$ decay of the purity. In Fig. 22 we fix $\hbar$ and change the coupling strength $\delta$ instead. Apart from oscillations we see a good agreement with the theory also for large $\delta$. Oscillations for times $t<10$ are a consequence of the fact that the time averaging of $V$ (2) converges only after some averaging time $t_{\text {ave }}$ which is of order $\sim 10$ in our case.

As for the second numerical example we take a $2+2$ DOF system $\left(d_{\mathrm{A}, \mathrm{B}}=2\right)$ which is the simplest case where 


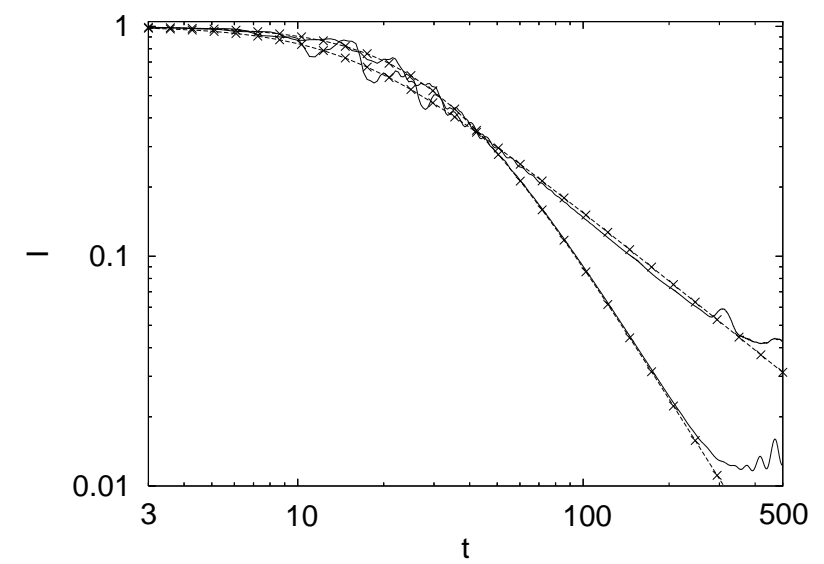

FIG. 3: Purity decay for a $2+2$ DOF system (17) and two different couplings showing different asymptotic power law decay. Full line is the numerics while the two dashed lines with crosses are theoretical predictions, the one with a smaller slope for (19) and the other for (18).

we can find different power of the asymptotic decay, depending on the topology of the coupling. The uncoupled Hamiltonian now reads

$$
\begin{aligned}
H_{0} & =\gamma_{1}\left(\hbar a_{1}^{+} a_{1}-\Delta\right)\left(\hbar a_{2}^{+} a_{2}-\Delta\right)+ \\
& +\gamma_{2}\left(\hbar a_{3}^{+} a_{3}-\Delta\right)\left(\hbar a_{4}^{+} a_{4}-\Delta\right) .
\end{aligned}
$$

Subscripts " 1 " and " 2 " describe two DOF of the subsystem "A", while " 3 " and "4" compose the subsystem "B". The parameters are $\gamma_{1}=1, \gamma_{2}=0.64$ and $\Delta=1.2$. The initial state is a product state of four boson coherent states, $\left|\psi_{1,2,3,4}(0)\right\rangle=|\alpha\rangle$, all with the same $\alpha=\sqrt{j^{*} / \hbar}$. For the coupling we consider two cases which will give different power of the asymptotic decay. Case I.: $V=V_{13}+V_{24}$, where two coupling terms are of the same form as for $1+1$ DOF system (13) and the indices denote between which two degrees of freedom the coupling acts. The matrix $u$ as well as the relevant determinant is easily calculated resulting in a simple expression for the purity (11)

$$
I(t)=\frac{1}{1+\left(8 j^{*} \delta t\right)^{2}}
$$

We see that we have a quadratic asymptotic decay, $I(t) \asymp$ $1 /(\delta t)^{2}$. Case II.: All to all coupling, $V=V_{13}+V_{14}+$ $V_{23}+V_{24}$, results in a rank-one $(r=1)$ matrix $u$ giving the purity decay (11)

$$
I(t)=\frac{1}{\sqrt{1+\left(16 j^{*} \delta t\right)^{2}}} .
$$

Results of the numerical simulation for both cases are shown in Fig. 3 The coupling strength and the location of the initial packets are $\delta=0.04, j^{*}=0.1$ for the case $I .$, and $\delta=0.02, j^{*}=0.2$ for the case II. From Fig. 3 we see that one indeed has asymptotic $t^{-1}$ or $t^{-2}$ decay, depending on the topology of the coupling.

In conclusion, we have derived purity decay for initial localized wave-packets in bipartite systems with a non-vanishing (non-trivial) time-averaged coupling operator. Such situation naturally occurs in systems where an uncoupled part of the Hamiltonian represents regular dynamics. Purity decays in time inversely proportional to the coupling strength and is independent of Planck's constant. The decay is algebraic with the asymptotic power-law exponent ranging between 1 and the minimal dimension of the subsystems depending on the topology of the coupling.

We thank Thomas H. Seligman for fruitful discussions. Financial supports by the grant P1-044 of the Ministry of Education, Science and Sports of Slovenia, and in part by the ARO grant (USA) DAAD 19-02-1-0086, and hospitality of $\mathrm{CiC}$ (Cuernavaca, Mexico), where parts of this work have been completed, are gratefully acknowledged.
[1] M. Brune et al., Phys. Rev. Lett. 77, 4887 (1996); L. Davidovich et. al., Phys. Rev. A 53, 1295 (1996).

[2] R. P. Feynman and F. L. Vernon, Ann. Phys. (N. Y.) 24, 118 (1963); A. O. Caldeira and A. J. Leggett, Physica 121A, 587 (1983).

[3] D. Braun et al., Phys. Rev. Lett. 86, 2913 (2001); W. T. Strunz et. al., Phys. Rev. A 67, 022101 (2003); W. T. Strunz and F. Haake, ibid. 67, 022102 (2003).

[4] Ph. Jacquod, Phys. Rev. Lett. 92, 150403 (2004).

[5] K. Furuya et al., Phys. Rev. Lett. 80, 5524 (1998); H. Fujisaki et al., Phys. Rev. E 67, 066201 (2003); J. Gong and P. Brumer, Phys. Rev. Lett. 90, 050402 (2003); R. M. Angelo et al., preprint quant-ph/0402091

[6] The special case $\bar{V}=0$ corresponding to freeze of fidelity decay [7] will be considered elsewhere. Such situation occurs in the case of quantum ergodicity, or in cases of peculiar geometric or dynamic symmetries.
[7] T. Prosen and M. Žnidarič, New. J. Phys. 5, 109 (2003); preprint quant-ph/0401142

[8] T. Prosen and T. H. Seligman, J. Phys. A 35, 4707 (2002).

[9] I. Bialynicki-Birula et al., Ann. Phys. (N.Y.) 51, 187 (1969).

[10] T. Prosen and M. Žnidarič, J. Phys. A 35, 1455 (2002).

[11] M. Žnidarič, Ph.D. Thesis, University of Ljubljana (2004), available as quant-ph/0406124

[12] T. Prosen, T. H. Seligman and M. Znidarič, Phys. Rev. A 67, 042112 (2003).

[13] M. Žnidarič and T. Prosen, J. Phys. A 36, 2463 (2003).

[14] W. H. Zurek, Phys. Today 44, 36 Oct. (1991).

[15] The very same expression holds also for a generalization of purity to echo dynamics, so-called echo purity (or purity fidelity), first used in [8]. 